\title{
Prevalence and drug resistance of mycobacteria in Turkish cystic fibrosis patients
}

\author{
Dilek Satana', Gonca Erkose-Genc ${ }^{1 *}$, Zeynep Tamay², Meltem Uzun, Nermin Guler and Zayre Erturan ${ }^{1}$
}

\begin{abstract}
Background: Isolation of mycobacteria in cystic fibrosis (CF) patients is increasingly being reported. Because of having long term antimicrobial treatment, CF patients are at risk of pulmonary infection with especially resistant nontuberculous mycobacteria (NTM) strains. The aim of the present study is to determine the prevalence of mycobacterium spp. and antimicrobial susceptibility in Turkish CF patients.

Methods: During a 5.5 year study period, 376 sputa from 130 CF patients were analyzed. Antimycobacterial susceptibility testing was performed by the Bactec 460 TB System and the E test method.

Results: Totaly 28 (7.44\%) Mycobacterium spp. were isolated from eight (6.15\%) CF patients. Five isolates (17.9\%) were identified as Mycobacterium tuberculosis complex (MTBC), 14 (50\%) as Mycobacterium abscessus and nine (32.1\%) as Mycobacterium lentiflavum. All MTBC isolates were found to be susceptible to streptomycin, isoniazid, rifampicin, and ethambutol. Resistance to some antibiotics was detected in some NTM strains. These are the first data about the prevalence of mycobacteria in CF patients from Turkey.
\end{abstract}

Conclusions: In pediatric CF patients, specific mycobacterial analysis of sputum specimens and susceptibility testing should be performed for allowing early detection, identification and the possibility of eradication of these bacteria.

Keywords: Mycobacteria, Nontuberculous mycobacteria, Mycobacterium tuberculosis complex, Cystic fibrosis, Drug resistance

\section{Background}

Cystic fibrosis (CF) is an autosomal recessive disease which defects in the Cystic Fibrosis Transmembrane Conductance Regulator (CFTR) gene product result in abnormally viscous secretions, mucus plugging of the airways, intense inflammation, chronic airway infection, and early death due to progressive bronchiectatic lung disease [1].

Infection with Mycobacterium tuberculosis complex (MTBC) strains in this patient group is rarely reported (1.34-3.41\%) [2-8]. These patients are at an increased risk of pulmonary colonization with opportunistic microorganisms. Nontuberculous mycobacteria (NTM) are increasingly isolated from patients with $\mathrm{CF}$, although the clinical significance of NTM in this population is not yet entirely understood [1]. It is not known whether these organisms are transmitted from person to person, acquired from

\footnotetext{
* Correspondence: goncaerkose@yahoo.com

${ }^{1}$ Istanbul Faculty of Medicine, Department of Medical Microbiology, Istanbul University, Istanbul 34093, Turkey

Full list of author information is available at the end of the article
}

environmental sources or obtained nosocomial [9]. Person-to-person transmission of NTM has been considered unlikely. However Aitken et al. [10] recently reported an outbreak of Mycobacterium abscessus ss massiliense in five cases with CF. The underlying structural airway disease and altered mucociliary clearance may be the reasons for the high prevalence of NTM in this patient group. Potential risk factors for NTM colonization/infection in CF include steroid treatment of allergic broncho-pulmonary aspergillosis and the use of bronchoscopes, aerosolized medications and tap water contaminated with NTM [9].

Multiple centers all over the world have reported a prevalence ranging from $1.09 \%$ to $22.7 \%$ for NTM in respiratory specimens of CF patients [3,6,7,11-16]. Oliver et al. [11] prospectively studied $986 \mathrm{CF}$ patients between $10-51$ years of age and found a prevalence of $13 \%$. The author reported that Mycobacterium avium complex (72\%) and Mycobacterium abscessus (16\%) were the most common species. An increased isolation rate of $M$. abscessus from CF patients has been reported in several 
countries [6,11,13-15,17,18]. The American Thoracic Society (ATS) published guidelines about the diagnosis and treatment of lung disease caused by NTM [9]. To meet criteria for NTM infection, patients must have compatible clinical findings, compatible radiographic findings, and bacteriologic findings including three positive cultures or two positive cultures and a positive smear for acid-fast bacteria. The first two criteria have been difficult to apply to patients with CF because of their longstanding respiratory symptoms, infective exacerbations, pre-existing radiographic abnormalities and systemic symptoms [3,9]. The ATS microbiologic criteria related to this subject, require the following; 1 . Positive culture results from at least two separate sputum samples or 2. Positive culture result from at least one bronchial wash or lavage or 3 . One or more sputum or bronchial washings that are culture positive for NTM if mycobacterial histopathologic features were evident [9].

Because of the risk of increased resistance due to long term antimicrobial treatment of common CF pathogens, susceptibility testing is recommended for NTM isolates from these patients [19]. M. abscessus, which is one of the most often isolated mycobacteria from CF patients, is reported to be resistant to most of the antimycobacterials including tetracyclines, fluoroquinolones, and sulphonamide [20,21]. Mussaffi et al. [13] reported six CF patients with NTM pulmonary disease, five of them had positive cultures for $M$. abscessus and one had a positive culture for $M$. simiae. All six cases were infected with multiresistant strains. The researchers observed that even when the NTM was susceptible initially, resistance developed rapidly after institution of therapy. In another study, M. chelonei and $M$. fortuitum were isolated from sputa of two different CF patients and found to be resistant to all drugs tested [3].

There is only two reports about multidrug resistant tuberculosis in this patient group. Two M. tuberculosis strains which were isolated from two patients with pulmonary infection, found to be resistant to all antibiotics $[2,8]$.

The prevalence of mycobacteria in Turkish CF patients is not known. The aim of the present study is to reveal the prevalence of MTBC and NTM isolates in Turkish CF patients and to determine the antimicrobial susceptibility of isolated species.

\section{Methods}

During a 5.5 year study period from April 2003 until November 2008, sputa which were sent to the Istanbul Faculty of Medicine, Department of Medical Microbiology for routine bacteriological or specific mycobacteriological culture were analyzed. The approval of an ethics committee was not necessary, because the clinical samples of the study were taken as part of standard patient care. Informed consent was given by all patients in this study. During this period a total of 376 respiratory specimens were collected from 130 patients.

Specimens were processed according to the standard protocols [22]. All sputa were decontaminated by $\mathrm{N}$-acetyl-L cysteine (NALC)/ sodium hydroxide $(\mathrm{NaOH})$ and concentrated by centrifugation (3800 g for $15 \mathrm{~min}$ at +4).

Processed specimens were prepared and stained by the Ziehl-Neelsen (ZN) method. The sediment was resuspended in $1 \mathrm{ml}$ phosphate buffer ( $\mathrm{pH}$ 6.8) and $0.4 \mathrm{ml}$ was inoculated into Bactec $12 \mathrm{~B}$ medium supplemented with antibiotics (Becton Dickinson, Spark, MD, USA) and onto Lowenstein-Jensen medium (Becton Dickinson, Spark, MD, USA). All cultures were incubated at $37^{\circ} \mathrm{C}$ for 10 weeks. The slants were examined twice weekly for two weeks and then weekly for a further 8 weeks. The Bactec 460 TB vials were controlled every 2 days during the first week and weekly thereafter.

$\mathrm{ZN}$ stained smears were prepared from positive Bactec 460 TB vials or LJ cultures. All acid-fast bacilli were identified to species level by the GenoType Mycobacterium CM/AS (Hain Lifescience GmbH, Nehren, Germany) assay.

Antimycobacterial susceptibility testing for MTBC isolates were performed by the Bactec 460 TB System. The critical concentrations used for the determination of resistance to major antimycobacterial agents were as follows: rifampicin $2 \mu \mathrm{g} / \mathrm{ml}$, isoniazid $0.1 \mu \mathrm{g} / \mathrm{ml}$, ethambutol $2.5 \mu \mathrm{g} / \mathrm{ml}$, and streptomycin $2 \mu \mathrm{g} / \mathrm{ml}$ [22].

Susceptibility testing for NTM isolates was performed by the E test method according to the manufacturer's instructions to clarithromycin, tigecycline, linezolid, amikacin, trimethoprim-sulfamethoxazole, doxycycline, cefoxitin, ciprofloxacin, imipenem, and tobramycin. The MIC values were evaluated according to the Clinical and Laboratory Standards Institute. The reference strain Staphylococcus aureus ATCC 29213 was used for E test, and H37Rv ATCC 27294 was used for the Bactec 460 TB System as quality control strains.

\section{Results}

A total of 376 sputa from 130 CF patients were investigated over a period of 5.5 years. The mean age of the patients was $12.1 \pm 3.1$ years (range: $5-17$ ). Out of 376 samples, a total of 28 (7.44\%) Mycobacterium spp. were isolated from eight (6.15\%) CF patients. Characteristics, culture and smear results of Mycobacterium spp. positive patients are given in Table 1. Five isolates (17.9\%) were identified as MTBC, 14 (50\%) as $M$. abscessus and nine (32.1\%) as $M$. lentiflavum. The isolation rate according to the patients was $3.07 \%$ (4/130) for MTBC and NTM strains each. Five MTBC strains were isolated from four different patients (two times from one patient), 14 M. abscessus strains were isolated from three different patients at different times, and nine $M$. lentiflavum strains were isolated from the same 
Table 1 Characteristics, culture and smear results of Mycobacterium spp. positive patients

\begin{tabular}{|c|c|c|c|c|}
\hline \multirow[t]{2}{*}{ Patient } & \multirow[t]{2}{*}{ Sex } & \multirow{2}{*}{$\begin{array}{c}\text { Smear } \\
(\mathrm{ZN})\end{array}$} & \multicolumn{2}{|l|}{ Culture results } \\
\hline & & & Mycobacterium spp. (n) & Co-pathogens \\
\hline 1 & M & + & MTBC (2) & Staphylococcus aureus \\
\hline 2 & $\mathrm{~F}$ & - & MTBC (1) & Stenotrophomonas maltophilia, Staphylococcus aureus, Pseudomonas aeruginosa \\
\hline 3 & M & - & MTBC (1) & Stenotrophomonas maltophilia, Escherichia coli, Enterobacter spp. \\
\hline 4 & $\mathrm{~F}$ & - & MTBC (1) & Not detected \\
\hline 5 & M & + & M. lentiflavum (9) & $\begin{array}{l}\text { Stenotrophomonas maltophilia, Staphylococcus aureus, Pseudomonas aeruginosa, } \\
\text { Aspergillus fumigatus }\end{array}$ \\
\hline 6 & $\mathrm{~F}$ & + & M. abscessus (10) & Stenotrophomonas maltophilia, Pseudomonas aeruginosa, Aspergillus fumigatus \\
\hline 7 & $\mathrm{~F}$ & + & M. abscessus (3) & Pseudomonas aeruginosa \\
\hline 8 & $\mathrm{~F}$ & - & M. abscessus (1) & Pseudomonas aeruginosa \\
\hline
\end{tabular}

M: Male, F: Female, MTBC: Mycobacterium tuberculosis complex.

patient at different times. Three of four (75\%) NTM positive and one of four (25\%) MTBC positive patients were also smear positive. The contamination rate of cultures was 9.8\% (37/376). Co-pathogens isolated together with Mycobacterium spp. were Pseudomonas aeruginosa $(\mathrm{n}=5)$, S. aureus $(\mathrm{n}=4)$, Stenotrophomonas maltophilia $(\mathrm{n}=4)$, and Aspergillus fumigatus $(\mathrm{n}=2)$. The characteristics of all patients, smear results and the distribution of co-colonizing bacteria are given in Table 2 .

All MTBC isolates were found to be susceptible to streptomycin, isoniazid, rifampicin, and ethambutol.

All $M$. abscessus isolates were susceptible to clarithromycin (MICs $0.125-1 \mu \mathrm{g} / \mathrm{ml}$ ) and tigecycline (MICs $0.19-0.5 \mu \mathrm{g} / \mathrm{ml}$ ). Eleven $(78.6 \%)$ of 14 strains were determined as susceptible to linezolid (MICs $4 \mu \mathrm{g} / \mathrm{ml}$ ), while the remaining three $(21.4 \%)$ were resistant (MICs $>256 \mu \mathrm{g} / \mathrm{ml}$ ). One strain $(7.1 \%$ ) was found to be resistant to amikacin (MIC $>256 \mu \mathrm{g} / \mathrm{ml}$ ) and 13 strains (92.8\%) showed intermediate MICs $(32-48 \mu \mathrm{g} / \mathrm{ml})$. All M. abscessus isolates were resistant to trimethoprim-sulfamethoxazole (MICs $>32 \mu \mathrm{g} / \mathrm{ml}$ ), doxycycline (MICs $>256 \mu \mathrm{g} / \mathrm{ml}$ ), cefoxitin (MICs $>256 \mu \mathrm{g} / \mathrm{ml}$ ),

Table 2 The characteristics of patients and the distribution of co-colonizing bacteria

\begin{tabular}{lccc}
\hline Characteristic & $\begin{array}{c}\text { MTBC and } \\
\text { NTM (-) }\end{array}$ & MTBC (+) & NTM (+) \\
\hline Total ( $\mathrm{n}=130)$ & 122 & 4 & 4 \\
Female (\%) & $58(47.5)$ & $2(50)$ & $3(75)$ \\
Mean age (years) & $12.1 \pm 3.2$ & $11.5 \pm 1.2$ & $15.5 \pm 1.7$ \\
Pseudomonas aeruginosa (\%) & 26.51 & - & 100 \\
Staphylococcus aureus (\%) & 28.03 & 50 & 50 \\
Stenotrophomonas maltophilia (\%) & 10.10 & 50 & 50 \\
Haemophilus influenza (\%) & 8.83 & - & - \\
Aspergillus species (\%) & 8.08 & - & 50 \\
Streptococcus pneumoniae (\%) & 4.54 & - & - \\
Klebsiella pneumonia (\%) & 1.51 & - & - \\
\hline
\end{tabular}

ciprofloxacin (MICs $>32 \mu \mathrm{g} / \mathrm{ml}$ ), imipenem (MICs $>32$ $\mu \mathrm{g} / \mathrm{ml}$ ) and tobramycin (MICs $>256-64 \mu \mathrm{g} / \mathrm{ml}$ ).

All of the M. lentiflavum isolates were susceptible to clarithromycin (MICs $0.25 \mu \mathrm{g} / \mathrm{ml}$ ), amikacin (MICs $12 \mu \mathrm{g} / \mathrm{ml}$ ) and ciprofloxacin (MICs $0.032 \mu \mathrm{g} / \mathrm{ml}$ ), and resistant to streptomycin $(2 \mu \mathrm{g} / \mathrm{ml})$, isoniazid $(0.1 \mu \mathrm{g} / \mathrm{ml})$, rifampicin $(2 \mu \mathrm{g} / \mathrm{ml})$, ethambutol $(2.5 \mu \mathrm{g} / \mathrm{ml})$, imipenem (MICs $>32 \mu \mathrm{g} / \mathrm{ml}$ ), tigecycline (MICs $>256 \mu \mathrm{g} / \mathrm{ml}$ ), cefoxitin (MICs $>256 \mu \mathrm{g} / \mathrm{ml}$ ), doxycycline (MICs $128 \mu \mathrm{g} / \mathrm{ml}$ ), and tobramycin (MICs $64 \mu \mathrm{g} / \mathrm{ml}$ ).

\section{Discussion}

There is little information about pulmonary tuberculosis in CF patients, and only a few cases have been published [2-8]. The isolation rates of MTBC from respiratory samples of these patients were reported as $1.34-3.41 \%[3,6,7]$.

These are the first data about the prevalence of mycobacteria in CF patients from Turkey. In our study, MTBC was isolated from sputa of four out of 130 patients (3.07\%). This rate is similar to the results reported from other studies.

NTM have emerged as new pathogens in CF patients recently [23]. CF centers worldwide have reported isolation rates ranging from $1.09 \%$ to $22.7 \%[7,12,13,15,16,19,24-27]$.

We found that during the study period $3.07 \%(4 / 130)$ of Turkish CF patients had NTM in their sputum samples. Three of these patients (75\%) had positive smears, and met the ATS microbiologic criteria [9].

Recovery of NTM from sputa of CF patients is usually hampered by the presence of other bacteria, especially $P$. aeruginosa, which is able to survive after routine sputum decontamination using NALC-NaOH. The Centers for Disease Control and Prevention recommend $5 \%$ oxalic acid (OxA) as decontaminating agent for $\mathrm{CF}$ specimens [28]. Whittier at al. [29] showed that using 5\% OxA after NALC-NaOH resulted with decrease in contamination rates and increase in recovery of mycobacteria. However Bange et al. [30] reported that although the contamination rate was reduced with NALC-NaOH-OxA, 
the overall sensitivity of mycobacterial recovery remained the same. In addition, Radhakrishnan et al. [19] pointed out that decontamination with NALC-NaOH-OxA may be too harsh and eliminate NTM in smear negative samples with lower numbers of mycobacteria. In an multicenter investigation Whittier et al. [28] concluded that although with the NALC-NaOH-OxA method NTM were successfully recovered from samples with AFB smear scores of $3+/ 4+$, but this was problematic in low inoculum samples with AFB smear scores of $1+/ 2+$. In the current study, only NALC-NaOH was used as decontaminating agents. Three of four (75\%) NTM positive and one of four (25\%) MTBC positive samples were smear positive with scores of $1+/ 2+$. Using OxA could have resulted with decrease in recovery rates of mycobacteria.

M. abscessus is a rapid growing mycobacterium (RGM) which is reported as a causative agent of respiratory disease in patients both with and without predisposing conditions, and cutaneous disease usually following accidental trauma or surgery [9]. It is one of the NTM that are most commonly isolated from CF patients, especially at younger ages $[9,13,20]$. M. abscessus isolates are uniformly resistant to standard antituberculous agents and infection with these microorganisms is extremely difficult to treat. Antibiotic susceptibility testing of clinically significant isolates is recommended due to variable in vitro drug susceptibilities to some drugs. Acquired mutational resistance to clarithromycin and amikacin can occur $[9,31]$.

The isolation rate of $M$. abscessus from pulmonary samples of CF patients was reported as 1.1-14.1\% [11-14,25,26]. Reported rates of pulmonary infection with these species according to the ATS microbiologic criteria was $1.5-3.8 \%[13,15,17,24,27]$.

In the current study, $M$. abscessus was the most commonly isolated NTM species (60.9\% of NTM species). $M$. abscessus was isolated from three patients with an isolation rate of $2.3 \%$, and two of these patients $(1.5 \%)$ met the ATS microbiologic criteria (positive culture results from at least two separate sputum samples) [9]. Although the isolation rate of this species from sputa of Turkish CF patients is low, it is in concordance with results reported from other studies.

M. lentiflavum is placed between the most commonly isolated newly described NTM species from clinical samples [32]. It was reported as the agent of cervical lymphadenitis mostly in children, skin and invasive infections, and a few respiratory tract infections have been detected in both immunocompetent and immunocompromised patients [32-34]. Because of its low virulence, the isolation of this species does not usually refer to an infection [32]. Immunosupression is a risk factor for infection with M. lentiflavum [33]. Resistance to primer antituberculous drugs is commonly reported [32-35].
There are a few studies which reported the isolation of M. lentiflavum from respiratory samples of CF patients with a rate of $0.06-20 \%[6,11,15,17]$. However, none of these studies reported a case which meets the ATS criteria for pulmonary infection.

In this study, M. lentiflavum was isolated from one patient's $(0.8 \%)$ nine separate samples, and this patient met the ATS microbiologic criteria. M. lentiflavum was consisted of $39.1 \%$ of NTM species isolated in the current study. The isolation rate of this species is similar to the rate which was reported in the other studies.

Oliver et al. [11] compared CF patients with and without NTM and found that culture-positive subjects had a higher frequency of $S$. aureus and a lower frequency of $P$. aeruginosa. They hypothesized that the presence of $S$. aureus may create conditions which can favor the presence of NTM in the airways. However Levy et al. [24] noted that there was no difference among the isolation rates of $S$. aureus from culture positive and negative patients regarding NTM. Interestingly, Musaffi et al. [13] and Levy et al. [24] reported that CF patients with NTM colonization and lung disease had higher frequency of $P$. aeruginosa. The same was observed in the current study. The rate of NTM and $S$. aureus co-colonization was $21.7 \%$, whereas the rate of NTM and P. aeruginosa co-colonization was higher as $34.7 \%$.

Long term use of macrolide in CF patients was shown to be a risk factor for NTM infection, especially with $M$. abscessus [24]. Renna et al. [36] showed that concentrations of azithromycin inhibited intracellular killing of mycobacteria in macrophage by impairing autophagic and phagosomal degradation and resulted in chronic infection in a mouse model. However, as there are some studies which have not established any association [19,26,37,38], the link between chronic macrolide use and NTM infection remains indeterminate.

In this study nine of the patients had long term macrolide therapy. Two of them (22.2\%) had infection with $M$. abscessus, one (11.1\%) had infection with $M$. lentiflavum, and one (11.1\%) other patient had infection with MTBC. As number of patients using macrolide is very small, the relationship between macrolide use and NTM infection could not get analyzed statistically.

In CF patients there is often poor correlation between in vitro drug susceptibility testing and clinical response for most antimicrobial drugs except for the macrolides. For Mycobacterium avium complex, susceptibility testing to macrolides is recommended because of the correlation with clinical response. Macrolide sensitivity testing and identification of the exact subspecies of $M$. abscessus also provides useful information for the treatment. Because of a deletion in the inducible erythromycin ribosome methyltransferase (erm) gene, M. abscessus subspecies massiliense is susceptible to 
macrolides, while $M$. abscessus ss abscessus and $M$. abscessus ss bolletii is resistant $[39,40]$.

There are only three studies which reported the drug susceptibility of MTBC strains which were isolated from CF patients. Asherova et al. [2] isolated two MTBC strains from two CF patients with pulmonary infection and found out that the two strains were resistant to all antibiotics tested. Smith et al. [3] isolated MTBC from sputa of three CF patients and reported that these three strains were susceptible to streptomycin, isoniazid, rifampicin, ethambutol, p-aminosalicylic acid, ethionamide, cycloserine, capreomycin, and kanamycin. Two of these strains were susceptible to pyrazinamide, while one strain was resistant. In the case report by Manika et al. [8] the strain was found resistant to streptomycin, isoniazid, rifampicin, ethambutol, pyrazinamide and quinolones, while it was susceptible to amikacin, kanamycin and capreomycin. In the current study all of MTBC isolates were found to be susceptible to streptomycin, isoniazid, rifampicin, and ethambutol.

M. abscessus is one of the NTM species which is most commonly isolated from CF patients and known to be the most pathogenic and chemotherapy-resistant RGM $[9,13,20]$. However there are only a few reports about the drug resistance of strains isolated from CF patients. Sanguinetti et al. [21] isolated M. abscessus from a CF patient which developed fatal disseminated infection. This strain was found to be resistant to streptomycin, isoniazid, rifampicin, ceftazidime, clarithromycin, levofloxacin, vancomycin, imipenem, amoxicillin, ethionamide, trimethoprim-sulfamethoxazole, ofloxacin, and amoxicillin-clavulanic acid. In another study, $M$. abscessus, which was the causative agent of a pulmonary infection in a CF patient, was initially susceptible to clarithromycin, ethambutol, amikacin, mefoxitin and developed in vitro resistance to all these drugs except for ethambutol after therapy [41]. Jönsson et al. [15] recovered a $M$. abscessus strain from a CF patient with persistent airway colonization, and detected that it was resistant to rifabutin, clarithromycin, clofazimine, and amikacin. In the current study all of the $M$. abscessus isolates were susceptible to clarithromycin and tigecycline. Eleven (78.6\%) of 14 strains were determined as susceptible to linezolid, while the remaining three $(21.4 \%)$ were resistant. One strain (7.1\%) was found to be resistant to amikacin and 13 strains (92.9\%) showed intermediate sensitivity pattern. All of $M$. abscessus isolates were resistant to trimethoprim-sulfamethoxazole, doxycycline, cefoxitin, ciprofloxacin, imipenem, and tobramycin.

Although the isolation of $M$. lentiflavum from respiratory samples of CF patients is reported in a few studies, there is no information about the susceptibility pattern of these strains $[6,11,15,17]$. In studies with $M$. lentiflavum strains isolated from non-CF patients, this species was usually found to be resistant to primary antituberculous drugs [32-35]. However, strains which are susceptible to clarithromycin, amikacin, ciprofloxacin, cycloserin, canamycin, and ofloxacin are reported [32,34,35]. In this study, all of $M$. lentiflavum isolates were susceptible to clarithromycin, amikacin, and ciprofloxacin, while they were fully resistant to streptomycin, isoniazid, rifampicin, ethambutol, imipenem, tigecycline, cefoxitin, doxycycline, and tobramycin.

\section{Conclusions}

Even though infection by MTBC in CF patients is rare, pulmonary infections due to NTM are increasingly being reported $[2-4,7,11,14]$. These are the first data about the prevalence of mycobacteria in CF patients from Turkey. We recovered MTBC from sputa of these patients with a rate of $3.07 \%$, and the isolation rate of NTM in this study is $3.07 \%$. In pediatric CF patients, specific mycobacterial analysis of sputum specimens are not routinely performed. In our opinion this should be done for allowing early detection, identification and the possibility of eradication of these bacteria. As these patients are under heavily antibiotic treatment, infection with resistant strains may occur. Because we detected resistance to some antibacterials in NTM strains, we propose that susceptibility testing should be performed for the NTM strains isolated.

\section{Competing interests}

The authors declare that no competing interest exists.

\section{Authors' contributions}

DS performed the experimental work and helped to draft the manuscript. GEG performed the experimental work and drafted the manuscript. ZT and NG carried out the clinical management of the patients. MU participated in the control and evaluation of the experiments. ZE designed the study and analyzed the data. All authors read and approved the final manuscript.

\section{Author details}

${ }^{1}$ Istanbul Faculty of Medicine, Department of Medical Microbiology, Istanbul University, Istanbul 34093, Turkey. ${ }^{2}$ Istanbul Faculty of Medicine, Department of Pediatrics, Istanbul University, Istanbul 34093, Turkey.

Received: 10 February 2014 Accepted: 5 June 2014

Published: 13 August 2014

\section{References}

1. LiPuma JJ: The changing microbiol epidemiology in cystic fibrosis. Clin Microbiol Rev 2010, 23:299-323.

2. Asherova IK, Feigelson J, Vasilyeva LA, Gabitov VJ: Cystic fibrosis complicated by multiresistant tuberculosis. Acta Paediatr 2006, 95:1513-1514

3. Smith MJ, Efthimiou J, Hodson ME, Batten JC: Mycobacterial isolations in young adults with cystic fibrosis. Thorax 1984, 39:369-375.

4. Morand PC, Burgel PR, Carlotti A, Desmazes-Dufeu N, Farhi D, Martin C, Kanaan R, Mangialavori L, Palangie E, Dusser D, Poyart C, Hubert D: Mediastinal tuberculosis in an adult patient with cystic fibrosis. $J$ Clin Microbiol 2011, 49:750-751.

5. Feigelson J, Delaisi B, Pecau Y, Kerzoncuf A, Anagnostopoulos C, Tournier G: Pneumopathie tuberculeuse au cours d'une mucoviscidose. Arch Pediatr 1997, 4:1209-1212. 
6. Somodevilla A, Domingo S, Agudo B, Hernandez M, Alonso M, Lopez: Mycobacterium in paediatric patients during a 2-year period in a health district of Madrid, Spain. Clin Microbiol Infect 2010, 16:31

7. Hjelte L, Petrini B, Kallenius G, Strandvik B: Prospective study of mycobacterial infections in patients with cystic fibrosis. Thorax 1990, 45:397-400.

8. Manika K, Giouleka P, Zarogoulidis K, Kioumis I: Multidrug-resistant tuberculosis in an adult with cystic fibrosis. Respir 2013, 85:350-353.

9. Griffith DE, Aksamit T, Brown-Elliott BA, Catanzaro A, Daley C, Gordin F, Holland SM, Horsburgh R, Huitt G, lademarco MF, Iseman M, Olivier K, Ruoss S, von Reyn CF, Wallace RJ Jr, Winthrop K: An official ATS/IDSA statement: Diagnosis, treatment, and prevention of nontuberculous mycobacterial diseases. Am J Respir Crit Care Med 2007, 175:367-416.

10. Aitken ML, Limaye A, Pottinger P, Whimbey E, Goss CH, Tonelli MR, Cangelosi GA, Dirac MA, Olivier KN, Brown-Elliott BA, McNulty S, Wallace RJ: Respiratory outbreak of Mycobacterium abscessus subspecies massiliense in a lung transplant and cystic fibrosis center. Am J Respir Crit Care Med 2012, 185:231-232.

11. Olivier KN, Weber DJ, Wallace RJ Jr, Faiz AR, Lee JH, Zhang Y, Brown-Elliot BA, Handler A, Wilson RW, Schechter MS, Edwards L, Chakraborti S, Knowles MR: Nontuberculous mycobacteria I: Multicenter prevalence study in cystic fibrosis. Am J Respir Crit Care Med 2003, 167:828-834.

12. Leitritz L, Griese M, Roggenkamp A, Geiger AM, Fingerle V, Heesemann J: Prospective study on nontuberculous mycobacteria in patients with and without cystic fibrosis. Med Microbiol Immunol 2004, 193:209-217.

13. Mussaffi H, Rivlin J, Shalit I, Ephros M, Blau H: Nontuberculous mycobacteria in cystic fibrosis associated with allergic bronchopulmonary aspergillosis and steroid therapy. Eur Respir J 2005, 25:324-328.

14. Gaillard JL: Mycobacterium abscessus and children with cystic fibrosis. Emerg Infect Dis 2003, 9:1587-1591.

15. Jönsson BE, Gilljam M, Lindblad A, Ridell M, Wold AE, Welinder-Olsson C: Molecular epidemiology of Mycobacterium abscessus, with focus on cystic fibrosis. J Clin Microbiol 2007, 45:1497-1504.

16. Seddon P, Fidler K, Raman S, Wyatt H, Ruiz G, Elston C, Perrin F, Gyi K, Bilton D, Drobniewski F, Newport M: Prevalence of nontuberculous mycobacteria in cystic fibrosis clinics, United Kingdom, 2009. Emerg Infect Dis 2013, 19:1128-1130.

17. Roux AL, Catherinot E, Ripoll F, Soismier N, Macheras E, Ravilly S, Bellis G, Vibet MA, Le Roux E, Lemonnier L, Gutierrez C, Vincent V, Fauroux B, Rottman M, Guillemot D, Gaillard JL, Herrmann JL: Multicenter study of prevalence of nontuberculous mycobacteria in patients with cystic fibrosis in France. J Clin Microbiol 2009, 47:4124-4128.

18. Russell CD, Claxton P, Doig C, Seagar AL, Rayner A, Laurenson IF: Non-tuberculous mycobacteria: a retrospective review of Scottish isolates from 2000 to 2010. Thorax. doi:10.1136/thoraxjn-2013-204260.

19. Radhakrishnan DK, Yau Y, Corey M, Richardson S, Chedore P, Jamieson F, Dell SD: Non-tuberculous mycobacteria in children with cystic fibrosis: Isolation, prevalence, and predictors. Pediatr Pulmonol 2009, 44:1100-1106.

20. Petrini B: Mycobacterium abscessus: An emerging rapid-growing potential pathogen. APMIS 2006, 114:319-328.

21. Sanguinetti M, Ardito F, Fiscarelli E, La Sorda M, D'Argenio P, Ricciotti G, Fadda G: Fatal pulmonary infection due to multidrug-resistant Mycobacterium abscessus in a patient with cystic fibrosis. $J$ Clin Microbiol 2001, 39:816-819.

22. Siddiqi SH: Bactec 460 TB System. Product and procedure manual, revision D. Sparks, MD: Becton Dickinson Microbiology Systems; 1995.

23. Ciofu O, Hansen CR, Hoiby N: Respiratory bacterial infections in cystic fibrosis. Curr Opin Pulm Med 2013, 19:251-258.

24. Levy I, Grisaru-Soen G, Lerner-Geva L, Kerem E, Blau H, Bentur L, Aviram M, Rivlin J, Picard E, Lavy A, Yahav Y, Rahav G: Multicenter cross-sectional study of nontuberculous mycobacterial infections among cystic fibrosis patients, Israel. Emerg Infect Dis 2008, 14:378-384.

25. Esther CR Jr, Esserman DA, Gilligan P, Kerr A, Noone PG: Chronic Mycobacterium abscessus infection and lung function decline in cystic fibrosis. JCF 2010, 9:117-123.

26. Giron RM, Maiz L, Barrio I, Martinez T, Salcedo A, Prados C: Nontuberculous mycobacterial infection in patients with cystic fibrosis: A multicenter prevalence study. Arch Bronconeumol 2008, 44:679-684.

27. Pierre-Audigier C, Ferroni A, Sermet-Gaudelus I, Le Bourgeois M, Offredo C, Vu-Thien H, Fauroux B, Mariani P, Munck A, Bingen E, Guillemot E, Quesne G, Vincent V, Berche P, Gaillard JL: Age-related prevalence and distribution of nontuberculous mycobacterial species among patients with cystic fibrosis. J Clin Microbiol 2005, 43:3467-3470.

28. Whittier S, Olivier K, Gilligan P, Knowles M, Della-Latta P, The Nontuberculous Mycobacteria in Cystic Fibrosis Study Group: The Nontuberculous mycobacteria in Cystic Fibrosis Study Group. Proficiency testing of clinical microbiology laboratories using modified decontamination procedures for detection of nontuberculous mycobacteria in sputum samples from cystic fibrosis patients. J Clin Microbiol 1997, 35:2706-2708.

29. Whittier S, Hopper RL, Knowles MR, Gilligan PH: Improved recovery of mycobacteria from respiratory secretions of patients with cystic fibrosis. J Clin Microbiol 1993, 31:861-864.

30. Bange FC, Kirschner R, Bottger EC: Recovery of mycobacteria from patients with cystic fibrosis. J Clin Microbio/ 1999, 37:3761-3763.

31. Haverkamp MH, van Wengen A, de Visser AW, van Kralingen KW, van Dissel JT, van de Vosse E: Pulmonary Mycobacterium abscessus: a canary in the cystic fibrosis coalmine. J Infect 2012, 64:609-612.

32. Tortoli E, Mattei R, Russo C, Scarparo C: Mycobacterium lentiflavum, an emerging pathogen? J Infect 2006, 52:185-187.

33. Tortoli E, Bartoloni A, Erba ML, Levre E, Lombardi N, Mantella A, Mecocci L: Human infections due to Mycobacterium lentiflavum. J Clin Microbiol 2002, 40:728-729.

34. Ibanez R, Serrano-Heranz R, Jimenez-Palop M, Roman C, Corteguera M, Jimenez S: Disseminated infection caused by slow-growing Mycobacterium lentiflavum. Eur J Clin Microbiol Infect Dis 2002, 21:691-692.

35. Philip J, Bhatia S, Sugar A, Berry N, Ruddy M: Mycobacterium lentiflavum-a cause of infections in the head and neck: case report and literature review. J Oral Maxillofac Surg 2011, 69:1114-1116.

36. Renna M, Schaffner C, Brown K, Shang S, Tamayo MH, Hegyi K, Grimsey NJ, Cusens D, Coulter S, Cooper J, Bowden AR, Newton SM, Kampmann B, Helm J, Jones A, Haworth CS, Basaraba RJ, DeGroote MA, Ordway DJ, Rubinsztein DC, Floto RA: Azithromycin blocks autophagy and may predispose cystic fibrosis patients to mycobacterial infection. J Clin Invest 2014, 121:3554-3563.

37. Verregghen M, Heijerman HG, Reijers M, van Ingen J, van der Ent CK: Risk factors for Mycobacterium abscessus infection in cystic fibrosis patients; a case-control study. J Cyst Fibros 2012, 11:340-343.

38. Catherinot E, Roux AL, Vibet MA, Bellis G, Ravilly $\mathrm{S}$, Lemonnier L, Le Roux E, Bernede-Bauduin C, Le Borgeois M, Herrmann JL, Guillemot D, Gaillard JL: Mycobacterium avium and Mycobacterium abscessus complex target distinct cystic fibrosis patient subpopulations. J Cyst Fibros 2013, 12:74-80.

39. Hill UG, Floto RA, Haworth CS: Non-tubeerculous mycobacteria in cystic fibrosis. J R Soc Med 2012, 105:14-18.

40. Leung JM, Olivier KN: Nontuberculous mycobacteria in patients with cystic fibrosis. Semin Respir Crit Care Med 2013, 34:124-134.

41. Forslöw U, Geborek A, Hjelte L, Petrini B, Heurlin N: Early chemotherapy for non-tuberculous mycobacterial infections in patients with cystic fibrosis. Acta Paediatr 2003, 92:910-915.

\section{doi:10.1186/1476-0711-13-28}

Cite this article as: Satana et al:: Prevalence and drug resistance of mycobacteria in Turkish cystic fibrosis patients. Annals of Clinical Microbiology and Antimicrobials 2014 13:28.

\section{Submit your next manuscript to BioMed Central and take full advantage of:}

- Convenient online submission

- Thorough peer review

- No space constraints or color figure charges

- Immediate publication on acceptance

- Inclusion in PubMed, CAS, Scopus and Google Scholar

- Research which is freely available for redistribution 